\title{
Help-seeking patterns and funding strategies in patients with pulmonary arterial hypertension on phosphodiesterase-5 inhibitors: an orphan disease with effective but costly treatment
}

Yinghao $\underline{\mathrm{Lim}}^{1}$, MBBS, MRCP, Siti Munawarah Bte $\underline{\text { Maaroof }}^{2}$, Ting Ting $\underline{\mathrm{Low}}^{1}$, MBBS, MRCP, Ivandito Kuntjoro ${ }^{1}$, MD, ABIM, James WL Yip ${ }^{1}$, MBBS, MRCP, Edgar Tay ${ }^{1}$, MBBS, MRCP

INTRODUCTION Pulmonary arterial hypertension (PAH) is associated with high medical and pharmaceutical costs. Phosphodiesterase type 5 (PDE5) inhibitors have been found to be beneficial but costly. They are not subsidised in Singapore except via the Medication Assistance Fund (MAF) Plus scheme. In this study, we described the help-seeking behaviour of patients and funding strategies for Singaporean patients on PDE5 inhibitors in our registry.

METHODS We consecutively recruited all patients with PAH who presented to our pulmonary hypertension specialty centre between 1 January 2003 and 29 December 2016. Singaporean patients on PDE5 inhibitors were included. Data recorded and analysed for this study included baseline demographics, whether the patients received MAF Plus funding, percentage of funding, and any additional source of subsidies.

RESULTS 114 (77.0\%) of 148 patients in the registry were Singapore citizens on PDE5 inhibitors. 75 (65.8\%) of these 114 patients had been seen by a medical social worker, of whom 16 were on MAF Plus funding. 14 of the remaining 59 patients were subsidised by MediFund, whereas the remainder were self-paying. 30 (26.3\%) patients in total were on some form of subsidy, and $28(24.6 \%)$ patients were on combination therapy. Of this group, nine were receiving MAF Plus subsidies. CONCLUSION Fewer than expected patients were found to be receiving drug subsidies for PAH. This was partly due to insufficient referrals and lack of requests for financial assistance. Patients on combination therapy had greater financial challenges. This study should spur us on to study funding gaps further and address them.

Keywords: Asia, healthcare financing, phosphodiesterase 5 inhibitors, pulmonary hypertension

\section{INTRODUCTION}

Pulmonary arterial hypertension $(\mathrm{PAH})$ is a rare and fatal disease. ${ }^{(1)}$ It is highly morbid and incurable. Recently, the availability of specific targeted therapies has resulted in improvements in patient outcomes. It has been shown that administering combination treatment at the onset may result in even better results. ${ }^{(2,3)}$ However, the cost of such therapy - which is often lifelong - is high.

Our institution maintains a registry of $\mathrm{PAH}$ patients that includes their clinical presentation, treatment and outcomes. Our previously published data shows that the majority of $\mathrm{PAH}$ patients in our institution are on phosphodiesterase type 5 (PDE5) inhibitors such as sildenafil and tadalafil.(4) PDE5 inhibitors have pulmonary vasodilatory effects and have been found to improve exercise capacity and haemodynamics in PAH patients. ${ }^{(5-7)}$ As such, they have been endorsed by international societies as a first-line therapeutic agent. ${ }^{(8)}$ The cost of treatment (per patient per month) in our institution is around SGD 500 for sildenafil and SGD 1,000 for tadalafil. PDE5 inhibitors are not on Singapore's standard drug list (SDL) and do not qualify for Medication Assistance Fund (MAF) subsidies. Patients who are unable to afford these medications are referred to a medical social worker to assess their financial needs, and alternative methods of funding are explored. One option that has helped many patients with the high costs is the MAF Plus scheme, which was put in place for patients on high-cost drugs that are not on the SDL or MAF list.

In this study, we aimed to describe the help-seeking behaviour of and funding strategies for Singaporean patients on PDE5 inhibitors in our registry.

\section{METHODS}

We consecutively recruited all patients with $\mathrm{PAH}$ who presented to our pulmonary hypertension $(\mathrm{PH})$ specialty centre between 1 January 2003 and 29 December 2016. The diagnosis of PAH was defined as mean pulmonary arterial pressure $>25 \mathrm{mmHg}$ at rest and pulmonary capillary wedge pressure $<15 \mathrm{mmHg}$ on right heart catheterisation, with exclusion of Groups 2, 3, 4 and $5 \mathrm{PH}$. Patients were enrolled if the clinical evidence was consistent with the accepted definition of $\mathrm{PAH}$ according to the managing PH specialist, even if the invasive haemodynamic data was incomplete. All enrolled patients had supportive evidence on echocardiography. Echocardiographic criteria were: (a) pulmonary arterial systolic pressure $>40 \mathrm{mmHg}$, estimated via tricuspid regurgitation jet; (b) features of right ventricular pressure overload with normal left ventricular systolic and diastolic function; and (c) normal left atrial volume. Patients

${ }^{1}$ Department of Cardiology, National University Heart Centre Singapore, ${ }^{2}$ Department of Medical Social Work, National University Hospital, Singapore Correspondence: Dr Lim Yinghao, Associate Consultant, Department of Cardiology, National University Heart Centre Singapore, NUHS Tower Block, Level 9, 1E Kent Ridge Road, Singapore 119228. yinghao_lim@nuhs.edu.sg 
with more than one contributor of $\mathrm{PH}$ were included if $\mathrm{PAH}$ was diagnosed to be the primary pathology. We decided on this approach for better generalisability to a real-world setting in which not all patients would have invasive haemodynamic data and often have multifactorial causes of PH. For this analysis, we restricted the study population to only patients on PDE5 inhibitors, as monotherapy or combination therapy. Foreigners and permanent residents were not included, as they are not eligible for local subsidies and are hence not representative of local PAH financing.

Data recorded and analysed for this study included the usage of drug therapy, whether the patients were receiving MAF Plus funding and their percentage of funding if they were, as well as any additional source of subsidies.

\section{RESULTS}

Out of a total of 148 patients in the registry, 114 (77.0\%) were Singapore citizens on PDE5 inhibitors, either as monotherapy or combination therapy. The mean age was 51.6 years with a $76.3 \%$ female predominance. The racial breakdown was consistent with our national census, with 71 (62.3\%) Chinese, 30 (26.3\%) Malay and 12 (10.5\%) Indian patients, as well as $1(0.9 \%)$ from the 'others' group. The most common aetiologies were congenital heart disease-associated PAH (CHD-PAH; 36.8\%), followed by idiopathic PAH (26.3\%) and connective tissue disease-associated PAH (CTD-PAH; 25.4\%). Other less common aetiologies were hereditary $\mathrm{PAH}$, portopulmonary $\mathrm{PAH}$ and pulmonary venoocclusive disease. Most cases (59.6\%) were prevalent, whereas the remainder were incident.

Most of our patients were symptomatic, the majority being Class II or III (37.7\% and $21.1 \%$, respectively) based on the New York Heart Association functional classification. The remaining haemodynamic and prognostic data is presented in Table I. The majority $(66.7 \%)$ of the patients were on sildenafil, whereas the remainder were on tadalafil. $75.4 \%$ of the patients were on monotherapy and the remainder on combination therapy, usually together with endothelin receptor antagonists such as bosentan or macitentan.

$75(65.8 \%)$ patients had been referred to a medical social worker (MSW). 16 (21.3\%) of these patients were eligible for MAF Plus funding: 14 were eligible for $75 \%$ funding, whereas two were eligible for $50 \%$ funding. The remaining drug costs were mainly paid for by the government endowment fund, MediFund, or an institutional endowment fund for heart patients, the Heart Fund. One patient also received partial funding from the Singapore Heart Foundation (SHF). Of the 59 patients who were previously reviewed by an MSW but were not receiving MAF Plus funding, 14 required assistance from MediFund. The remaining 45 patients paid for their own therapy (Fig. 1). Hence, $26.3 \%$ of the 114 patients on PDE5 inhibitors were on partial or complete funding. Of the 28 patients on combination therapy, 17 were known to an MSW. Of these, seven received $75 \%$ funding by MAF Plus, two received $50 \%$ funding and the remaining were self-paying (Fig. 2).
Table I. Baseline characteristics of Singaporean pulmonary arterial hypertension (PAH) patients on phosphodiesterase type 5 inhibitors $(n=114)$.

\begin{tabular}{|c|c|}
\hline Characteristic & No. (\%) \\
\hline Age $^{*}(y r)$ & 51.6 \\
\hline \multicolumn{2}{|l|}{ Gender } \\
\hline Female & $87(76.3)$ \\
\hline Male & $27(23.7)$ \\
\hline \multicolumn{2}{|l|}{ Ethnicity } \\
\hline Chinese & $71(62.3)$ \\
\hline Malay & $30(26.3)$ \\
\hline Indian & $12(10.5)$ \\
\hline Others & $1(0.9)$ \\
\hline \multicolumn{2}{|l|}{ Aetiology } \\
\hline CHD-PAH & $42(36.8)$ \\
\hline iPAH & $30(26.3)$ \\
\hline CTD-PAH & $29(25.4)$ \\
\hline Hereditary PAH & $6(5.3)$ \\
\hline Portopulmonary & $2(1.8)$ \\
\hline PVOD & $2(1.8)$ \\
\hline Others & $3(2.6)$ \\
\hline \multicolumn{2}{|l|}{ Onset } \\
\hline Incident & $46(40.4)$ \\
\hline Prevalent & $68(59.6)$ \\
\hline \multicolumn{2}{|l|}{ Functional class } \\
\hline 1 & $16(14.0)$ \\
\hline II & $43(37.7)$ \\
\hline III & $24(21.1)$ \\
\hline IV & $3(2.6)$ \\
\hline NA & $28(24.6)$ \\
\hline \multicolumn{2}{|l|}{ Haemodynamics* } \\
\hline $\mathrm{mPAP}(\mathrm{mmHg})$ & 48.9 \\
\hline Cardiac output (L/min) & 3.47 \\
\hline PVR (Wood units) & 12.9 \\
\hline mRAP $(\mathrm{mmHg})$ & 9.9 \\
\hline Systolic BP (mmHg) & 123.0 \\
\hline \multicolumn{2}{|l|}{ Other parameters } \\
\hline Creatinine $^{*}(\mathrm{~mL} / \mathrm{min})$ & 87.0 \\
\hline $6 \mathrm{MWD}^{*}(\mathrm{~m})$ & 328 \\
\hline NT-proBNP* $(p g / m L)$ & 2,492 \\
\hline REVEAL score* & 7.15 \\
\hline Pericardial effusion & $18(15.8)$ \\
\hline \multicolumn{2}{|l|}{ Therapy } \\
\hline Sildenafil & $76(66.7)$ \\
\hline Tadalafil & $38(33.3)$ \\
\hline Monotherapy & $86(75.4)$ \\
\hline Combination & $28(24.6)$ \\
\hline
\end{tabular}

*Data presented as mean. 6MWD: six-minute walk distance; BP: blood pressure; CHD-PAH: congenital heart disease-associated PAH; CTD-PAH: connective tissue disease-associated $\mathrm{PAH}$; $\mathrm{PAH}$ : idiopathic $\mathrm{PAH}$; mPAP: mean pulmonary artery pressure; mRAP: mean right atrial pressure; NT-proBNP: N-terminal prohormone brain natriuretic peptide; PVOD: pulmonary veno-occlusive disease; PVR: pulmonary vascular resistance. Haemodynamic parameters were obtained within $6 \pm 3$ months from the enrollment date. 


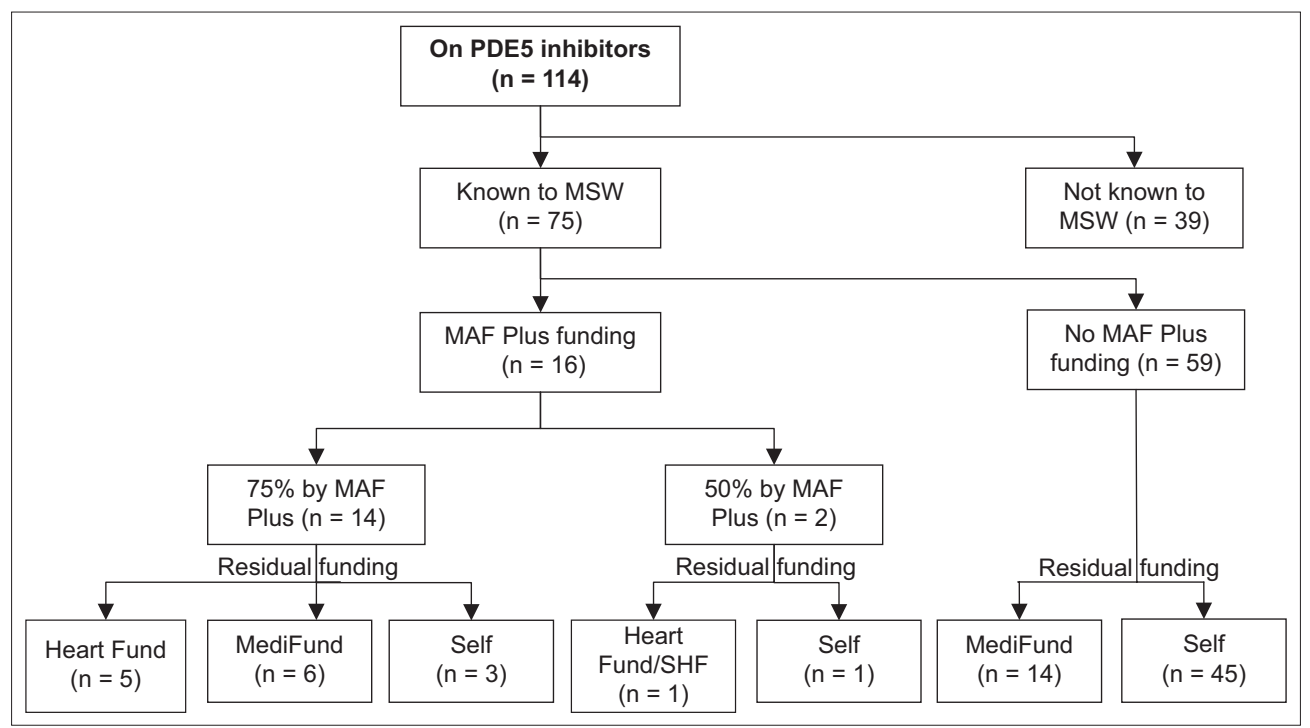

Fig. 1 Chart shows breakdown of funding for Singaporean pulmonary arterial hypertension patients on PDE5 inhibitors. MAF: Medication Assistance Fund; MSW: medical social worker; PDE5: phosphodiesterase type 5; SHF: Singapore Heart Foundation

\section{DISCUSSION}

$\mathrm{PAH}$ is a highly morbid condition, although recent medical advances have allowed for an expanded therapeutic armamentarium that includes PDE5 inhibitors, endothelin receptor antagonists, soluble guanylyl cyclase stimulators and prostacyclin analogues. ${ }^{(8)}$ The disease and its treatment has been associated with a substantive economic burden on patients and the healthcare system. A systematic review showed that estimated total healthcare costs ranged from USD 2,476 to USD 11,875 per patient per month, with pharmacy costs being an important driver. Sildenafil was reported to be cost-effective and associated with lower costs and higher efficacy. ${ }^{(9)}$

In our institution, the cost of PDE5 inhibitor therapy is estimated to be about SGD 500-1,000 per patient per month prior to funding. For patients on combination therapy, pharmacy costs are cumulative. This would be a significant burden for most Singaporeans who do not receive subsidies, considering that the median income per household member was SGD 2,500 in 2019. ${ }^{(10)}$

The high morbidity of $\mathrm{PAH}$ leads to high healthcare costs even after initiation of PAH therapy. A study of United Statesbased insurance claims showed that total healthcare costs did not change after initiating PAH therapy. The cost savings obtained from decreasing non-pharmacy health costs such as hospital admissions were made up for by the increased pharmacy costs of PAH drugs, which were USD 19,961 over a six-month period. ${ }^{(11)}$

Healthcare financing in Singapore is built on a tiered copayment model. The first tier is government subsidies. The next is the compulsory medical savings scheme MediSave, which is claimable for hospitalisation as well as selected outpatient chronic condition expenditure. The third tier is provided through a national health insurance scheme, MediShield Life, that covers hospitalisation and selected costly outpatient treatments such as dialysis and chemotherapy or radiotherapy. The last tier of funding is through MediFund, an endowment fund for patients who require additional financial assistance on top of the existing

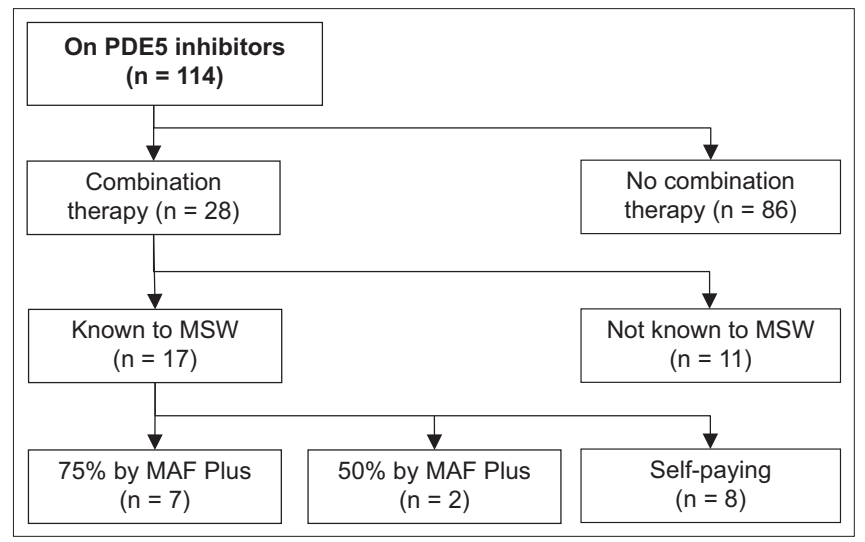

Fig. 2 Chart shows breakdown of funding for Singaporean pulmonary arterial hypertension patients on combination therapy. MAF: Medication Assistance Fund; MSW: medical social worker; PDE5: phosphodiesterase type 5

provisions. ${ }^{(12,13)}$ The Ministry of Health provides a blanket subsidy for all drugs on the SDL for patients served at subsidised specialist outpatient clinics and polyclinics, and can be up to $75 \%$ of the drug cost. The MAF was set up in 2010 to provide subsidies for newer or high-cost medications that are not on the SDL for specific indications. The SDL and MAF list are reviewed periodically by a drug advisory committee. ${ }^{(14-16)}$

PDE5 inhibitors are not MediSave claimable and are not on either of the drug subsidy lists. Hence, patients who need financial assistance have to apply for special funding through the MAF Plus scheme. The threshold criteria for qualification is an income per household member of SGD 2,600 or less, approval of the medication by the Health Sciences Authority, and the lack of a cheaper alternative. The MSW plays an integral role in financial assessment and the application process.

Our patients are similar in profile to those of other international registries, although idiopathic PAH is less common locally. They tend to present symptomatically with an intermediate risk profile. $^{(1,4)}$ This is significant, as there are growing international 
recommendations to initiate treatment with combination therapy for patients who are at intermediate or high risk. ${ }^{(8)}$

In the present study, only two-thirds of the patients were reviewed by an MSW. Of these patients, 59 (78.7\%) eventually did not receive MAF Plus funding. 14 of these patients already had MediFund assistance, which was sufficient to cover drug costs. Of the remaining 45 patients, 29 were assessed by a MSW prior to the inception of the MAF Plus scheme or were not reviewed for the purposes of financial assessment. A minority were not keen to go through the financial assessment process.

It is striking that only $26.3 \%$ of all patients on PDE5 inhibitors received at least a partial subsidy. Considering that the median income per household member in 2019 (SGD 2,500) was lower than the threshold for MAF Plus (SGD 2,600), our patients appeared to be utilising less subsidies than expected. In our analysis, we also noticed that one-third of our patients were not referred for MSW assessment for subsidies. Of those who were seen by an MSW but did not receive subsidies, most patients did not request for financial assistance, or were seen in an era in which MAF Plus was not available and were lost to follow-up thereafter.

Although our patients were in the intermediate risk category on average, only one-quarter of them were on combination therapy. $61 \%$ of these patients were seen by an MSW and over half of those assessed were on MAF Plus funding - with the majority being in a higher needs category on $75 \%$ funding. Given the high costs of PAH drugs, especially with multi-drug usage, the aforementioned costs reflect the additional financial challenges that patients on combination therapy face, and constitute a potential reason for the low uptake of combination therapy.

Furthermore, there is currently no system of routine MSW referrals upon diagnosis of $\mathrm{PAH}$ and initiation of therapy; instead, referrals occur when the patient expresses a need. The data presented suggests that routine referrals for financial assessment would be beneficial and could possibly increase the uptake of PAH therapy. Reviews for subsidies should be revisited periodically, especially as policies change.

These findings need to be explored in future studies to determine if there is a lack of awareness of the availability of financial assistance or a cultural reluctance in local patients to request for financial help. We can also further explore whether drug costs are a major obstacle to patient acceptance of PAH therapy.

This study was not without limitations. We were not able to obtain household financial details for individual patients in this study, which would have enabled us to understand if the patients who had not seen MSWs were those who had the financial means to pay on their own. Also, patient numbers were small and from a single academic centre. We were also unable to study clinical outcomes among the patients who received funding compared to those who did not receive funding.

In conclusion, $\mathrm{PAH}$ is a disease with significant morbidity. Drug therapy is efficacious but associated with high costs.
Although financial help is available, we identified that fewer patients received or accepted funding than expected, likely due to insufficient referrals to MSWs for drug subsidies. Even when they were referred, there was no follow-up request for financial assistance. Patients on combination therapy face additional financial challenges that could be a barrier to greater uptake of more effective therapy. The increasing number of agents with higher cost in the near future is likely to result in a more challenging financing environment, which needs to be planned for as soon as possible.

\section{REFERENCES}

1. McGoon MD, Benza RL, Escribano-Subias P, et al. Pulmonary arterial hypertension: epidemiology and registries. J Am Coll Cardiol 2013; 62(25 Suppl):D51-9.

2. Galiè N, Palazzini M, Manes A. Pulmonary arterial hypertension: from the kingdom of the near-dead to multiple clinical trial meta-analyses. Eur Heart J 2010; 31:2080-6.

3. Galiè N, Barberà JA, Frost $A E$, et al; $A M B I T I O N$ Investigators. Initial use of ambrisentan plus tadalafil in pulmonary arterial hypertension. New Engl J Med 2015; 379:834-44

4. Lim Y, Low TT, Chan SP, et al. Pulmonary arterial hypertension in a multi-ethnic Asian population: characteristics, survival and mortality predictors from a 14year follow-up study. Respirology 2019; 24:162-70. [Epub ahead of print]

5. Galiè N, Ghofrani HA, Torbicki A, et al; Sildenafil Use in Pulmonary Arterial Hypertension (SUPER) Study Group. Sildenafil citrate therapy for pulmonary arterial hypertension. N Engl J Med 2005; 353:2148-57.

6. Galiè N, Brundage BH, Ghofrani HA, et al; Pulmonary Arterial Hypertension and Response to Tadalafil (PHIRST) Study Group. Tadalafil therapy for pulmonary arterial hypertension. Circulation 2009; 119:2894-903.

7. Jing ZC, Yu ZX, Shen JY, et al; Efficacy and Safety of Vardenafil in the Treatment of Pulmonary Arterial Hypertension (EVALUATION) Study Group. Vardenafil in pulmonary arterial hypertension: a randomized, double-blind, placebocontrolled study. Am J Respir Crit Care Med 2011; 183:1723-9.

8. Galiè N, Humbert M, Vachiery JL, et al. 2015 ESC/ERS Guidelines for the diagnosis and treatment of pulmonary hypertension: the Joint Task Force for the Diagnosis and Treatment of Pulmonary Hypertension of the European Society of Cardiology (ESC) and the European Respiratory Society (ERS): Endorsed by: Association for European Paediatric and Congenital Cardiology (AEPC), International Society for Heart and Lung Transplantation (ISHLT). Eur Respir J 2015; 46:903-75.

9. Gu S, Hu H, Dong H. Systematic review of the economic burden of pulmonary arterial hypertension. Pharmacoeconomics 2016; 34:533-50.

10. Department of Statistics Singapore. Table 10A. Average and median monthly household income from work per household member (including employer CPF contributions) among resident and resident employed households, 20002019. Available at: https://www.tablebuilder.singstat.gov.sg/publicfacing/ createSpecialTable.action?refld=16744. Accessed February 24, 2020.

11. Burger CD, Ozbay AB, Lazarus HM, et al. Treatment patterns and associated health care costs before and after treatment initiation among pulmonary arterial hypertension patients in the United States. J Manag Care Spec Pharm 2018; 24:834-42.

12. Ministry of Health, Singapore. Costs and financing [online]. Available at: https:// www.healthhub.sg/a-z/costs-and-financing/5/costs_and_financing_overall. Accessed February 24, 2020.

13. Ministry of Health, Singapore. MediSave [online]. Available at: https://www. moh.gov.sg/cost-financing/healthcare-schemes-subsidies/medisave. Accessed February 24, 2020.

14. Ministry of Health, Singapore. The Straits Times. List of subsidised drugs reviewed regularly [online]. Available at: https://www.straitstimes.com/forum/letters-onthe-web/list-of-subsidised-drugs-reviewed-regularly. Accessed February 24, 2020 .

15. Ministry of Health, Singapore. Drug Subsidies \& Schemes [online]. Available at: https://www.moh.gov.sg/cost-financing/healthcare-schemes-subsidies/drugsubsidies-schemes. Accessed February 24, 2020.

16. Pwee Keng Ho, Deputy Director (Health Technology Assessment), Ministry of Health, Singapore. Health Technology Assessment \& Policy-Making: the Singapore Experience [online]. Available at: https://www.who.int/medical_ devices/02_keng_ho_pwee.pdf. Accessed February 24, 2020. 


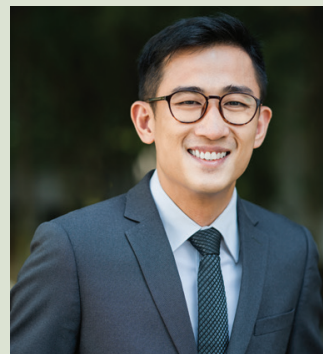

\section{About the First Author}

Dr Lim Yinghao is an Associate Consultant with the Department of Cardiology, National University Heart Centre, Singapore. His clinical interests are in structural heart disease, adult congenital heart disease, pulmonary hypertension and cardiac imaging. He performs structural and congenital heart intervention as well as interventional echocardiography. Additionally, he also performs transoesophageal echocardiography. Dr Lim is an established medical educator and is course co-director for the Chia Boon Lock Cardiology Review Course and Cardiology PACES Course. He is a core faculty member of the Cardiology Senior Residency Programme. His research interests are in pulmonary hypertension, structural heart disease as well as medical innovation. 